\title{
Aptitud combinatoria y potencial agronómico de líneas de maíz con diferente nivel de endogamia
}

Combining ability and agronomic potential of maize lines with different level of inbreeding
María de los Ángeles Acevedo-Cortés ${ }^{1}$ (D) Antonio Castillo-Gutiérrez ${ }^{1^{*}}$ (D) María Andrade-Rodríguez $^{1}$ (D), María Eugenia Nuñez-Valdez ${ }^{2}$ (D) , Francisco Perdomo-Roldan ${ }^{1}$ (iD, Ramón Suárez-Rodríguez ${ }^{3}$ (iD

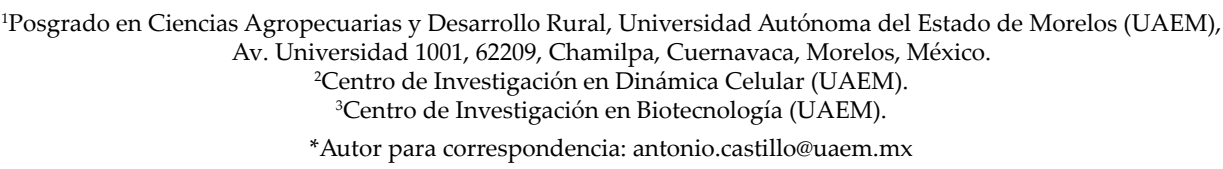

Fecha de recepción:

27 de abril de 2020

Fecha de aceptación:

23 de agosto de 2020

Disponible en línea:

18 de diciembre de 2020

Este es un artículo en acceso

abierto que se distribuye de acuerdo a los términos de la licencia Creative Commons.

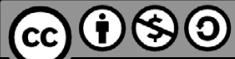

Reconocimiento-

NoComercial-

CompartirIgual 4.0

Internacional

\section{RESUMEN}

Determinar la aptitud combinatoria de líneas endogámicas de maíz es de suma importancia para su desarrollo, por lo cual, en este estudio, se evaluaron 15 mestizos en tres localidades de Morelos, México (P-V 2017); 36 cruzas dialélicas (O-I 2018/2019) en una sola localidad, así como 15 líneas endogámicas per se (P-V 2018). Los objetivos de dichas evaluaciones fueron estimar y seleccionar líneas con buena aptitud combinatoria a través de prueba de mestizos; determinar si las líneas de alta Aptitud Combinatoria General (ACG) positiva para rendimiento de grano forman cruzas de alta Aptitud Combinatoria Específica (ACE) positiva con líneas de ACG negativa, y evaluar el potencial agronómico de líneas de diferente nivel de endogamia. Las variables analizadas fueron la floración masculina, altura de planta, peso de 100 semillas y rendimiento. Las líneas CML341, CML576 y CML549 resultaron con ACG positiva en mestizos y cruzas dialélicas, y las CML340, CML342, CML341, LUM124 y LUM194 fueron las de mayor rendimiento de grano.

\section{PALABRAS CLAVE}

Mestizos, cruzas dialélicas, evaluación per se, Zea mays L.

\section{ABSTRACT}

In the development of inbred maize lines, it is of great importance to determine their combinatory aptitude; therefore, in this study, 15 top-crosses were evaluated in three localities from Morelos, Mexico (P-V 2017); 36 diallelic crosses were evaluated in a single locality (O-I 2018/2018/2019), and 15 inbred lines were evaluated per se (P-V 2018). The objectives were to estimate and select lines with good General Combinatory Aptitude (GCA) through topcrosses testing, to determine if positive high GCA lines for grain yield form positive high Specific Combinatory Aptitude (SCA) crosses with negative GCA lines, and to evaluate the agronomic potential of lines of different level of inbreeding. The variables studied were male flowering, plant height, weight of 100 kernels, and grain yield. The CML341, CML576 and CML549 lines resulted in positive GCA in topcrosses and diallelic crosses, whereas CML340, CML342, CML341, LUM124 and LUM194 were the highest in grain yield.

\section{KEYWORDS}

Top cross, diallelic crosses, evaluation per se, Zea mays L. 


\section{INTRODUCCIÓN}

En México, el maíz es uno de los cultivos de mayor importancia a nivel nacional, por lo que se producen anualmente alrededor de 7.5 millones de hectáreas, de las cuales, en el ciclo primavera-verano, se siembran aproximadamente 5.5 millones de hectáreas, con un rendimiento promedio de $2.6 \mathrm{t} \mathrm{ha}^{-1}$ (SIAP 2017); en el estado de Morelos, la superficie sembrada de maíz es de cerca de 33,412 ha, con un promedio de rendimiento de $3.7 \mathrm{t} \mathrm{ha}^{-1}$ (SAGARPA 2017). Debido a que los rendimientos de grano obtenidos en temporal son bajos, resulta necesario implementar estrategias que los incrementen, y una alternativa es el mejoramiento genético de este cereal.

En programas de mejoramiento genético de maíz, un objetivo importante es generar líneas endogámicas con alto potencial de rendimiento, buen comportamiento agronómico y buena aptitud combinatoria (Buenrostro-Robles et al. 2017). La aptitud combinatoria general (ACG) se asocia a la acción génica de tipo aditivo (Falconer y Mackay 2006), en tanto que la aptitud combinatoria especifica (ACE) es el resultado de la acción génica no aditiva (dominancia y epistasis) (Poehlman y Sleper 2003). En el fitomejoramiento, se han desarrollado técnicas para estimar la magnitud de los efectos de la ACG y ACE, de las cuales destacan la prueba basada en la formación y evaluación de mestizos, que facilita la estimación del valor genético aditivo del germoplasma (Sánchez-Ramírez et al. 2020), y la basada en cruzas dialélicas, que permite determinar tanto el valor aditivo como el no aditivo (Ramírez-Díaz et al. 2019).

La prueba de mestizos es comúnmente utilizada debido a la facilidad para efectuarla, y al costo bajo deimplementarla con un gran número de líneas endogámicas (Castañón et al. 1998); otra razón importante de su uso es el alto grado de efectividad para discriminar y seleccionar líneas con sobresaliente ACG (LedesmaMiramontes et al. 2015), tanto en pruebas tempranas como en pruebas tardías (Ramírez et al. 1998; Ramírez et al. 2007). La principal ventaja de la prueba temprana es que permite eliminar al menos 50\% de líneas con baja o nula ACG, lo que reduce costos de evaluación de un gran número de líneas en generaciones avanzadas (Sprague 1946). La prueba temprana se sustenta en que la aptitud combinatoria de las líneas se define en las primeras etapas de endogamia (Bernardo 1992), en tanto que la evaluación tardía se fundamenta en la selección de líneas en etapas tempranas, con base en la expresión fenotípica de características de importancia agronómica, para posteriormente formar mestizos e identificar las de mayor ACG (Ramírez et al. 1998). Por otro lado, un elemento clave en la prueba de mestizos es la elección del probador, lo que ha sido ampliamente estudiado, y se sugiere que debe poseer una baja expresión fenotípica de la característica de interés, así como una nula aptitud combinatoria (Hallauer et al. 2010; Lobato-Ortiz et al. 2010).

La prueba de mestizos formados con líneas $S_{5}$ y dos líneas del Centro Internacional de Mejoramiento de Maíz y Trigo (CIMMYT), como probadores, permitió seleccionar líneas de alta ACG en la variable rendimiento de grano $\left(\mathrm{kg} \mathrm{ha}^{-1}\right)$, valor que fluctuó entre 483 y $768 \mathrm{~kg} \mathrm{ha}^{-1}$ (Castañón et al. 1998). En otra evaluación de mestizos, se encontraron seis sobresalientes con rendimientos de grano de entre 7,215 y 7,816 kg ha-1, estadísticamente igual a los testigos comerciales usados (Velázquez-Cardelas et al. 2018). Por otro lado, se han reportado rendimientos de grano que oscilan entre los 4,495 y los $8,074 \mathrm{~kg} \mathrm{ha}^{-1}$, y un rendimiento del probador de 3,524 $\mathrm{kg} \mathrm{ha}^{-1}$ (LedesmaMiramontes et al. 2015).

En cuanto a la formación y evaluación de cruzas dialélicas, se utiliza frecuentemente para evaluación del potencial genético de líneas endogámicas (Hallauer et al. 2010; Sánchez-Ramírez et al. 2017). Al respecto, se reporta que las cruzas con efectos altos de ACE en rendimiento son el resultado de líneas progenitoras con alta ACG (Escorcia-Gutiérrez et al. 2010). Pérez-López et al. (2014) dieron a conocer que, en cruzas de alto rendimiento, al menos una de las líneas progenitoras presentó alta ACG y mostró efectos altos en ACE, mientras que cruzas cuyas dos líneas fueron de baja ACG presentaron efectos bajos de ACE. Otro estudio indicó que altos valores de ACE se deben a altos rendimientos de las cruzas y a valores bajos o negativos de la ACG de las líneas que intervienen en las cruzas, o a la combinación de una línea con alta ACG con otra de valores bajos o negativos de ACG (Wong-Romero et al. 2006). En otras investigaciones, se menciona que, con base en los efectos genéticos de las cruzas, se encontraron efectos altos y positivos para rendimiento cuando intervienen líneas tanto con alta ACG, como con valores bajos y negativos 
(Cervantes-Ortiz et al. 2018; Guillen-de la Cruz et al. 2009). También se han desarrollado análisis estadísticos para estimar la ACG y ACE en cruzas dialélicas; uno de ellos es el método IV de Griffing (1956), que evalúa únicamente las cruzas $\mathrm{F}_{1}$ directas.

Conocer el tipo de acción génica involucrada en la expresión de caracteres de interés es un factor que determina la utilidad futura y el potencial comercial de las líneas, ya sea para desarrollar híbridos (Akaogu et al. 2017; Hallauer et al. 2010; López et al. 2017; Rodríguez-Pérez et al. 2016) o sintéticos (Cervantes-Ortiz et al. 2018). En el programa de fitomejoramiento de maíz de la Escuela de Estudios Superiores de Xalostoc (EESuX), perteneciente a la Universidad Autónoma del Estado de Morelos (UAEM), se han desarrollado líneas tolerantes a sequía considerando el rendimiento per se, el corto intervalo antésis-emergencia de estigmas y el peso de semilla (Rebolloza et al. 2016); sin embargo, no se había realizado el estudio de la aptitud combinatoria, por lo que los principales objetivos de la presente investigación fueron: seleccionar líneas de maíz con buena aptitud combinatoria, a través de prueba de mestizos; determinar si las líneas de alta ACG positiva en rendimiento de grano pueden formar cruzas de alta ACE positiva con líneas de ACG negativa, y evaluar el potencial agronómico de las líneas de diferente nivel de endogamia, mediante cuatro características cuantitativas.

\section{Materiales y Métodos}

\section{Germoplasma base}

El germoplasma base del estudio está constituido por 15 líneas endogámicas de maíz tolerantes a sequía (Cuadro 1); de éstas, 10 pertenecen al programa de mejoramiento de la EESuX, dependiente de la UAEM, y 5 fueron proporcionadas por el Banco de Germoplasma del CIMMYT; adicionalmente, se utilizó la variedad experimental VUAT de baja ACG, desarrollada y facilitada por la Universidad Autónoma de Tamaulipas. Las 15 líneas se utilizaron para llevar a cabo un estudio sobre la ACG y ACE, así como para determinar el potencial agronómico de las líneas mediante evaluación per se, en tanto que la variedad VUAT se utilizó como probador genético del grupo de líneas.

\section{Formación y evaluación de mestizos}

La formación de mestizos se realizó en el ciclo agrícola de otoño-invierno 2016/2017, en el campo experimental de la EESuX, con instalaciones ubicadas en Ayala, Morelos, México (18 44' 29" N y $98^{\circ} 54^{\prime} 38^{\prime \prime}$ O, a una altitud de $1220 \mathrm{~m}$ ), para lo cual, en condiciones de riego, se sembraron las 15 líneas y la variedad experimental VUAT (probador) en un lote con aislamiento genético. La siembra del germoplasma se hizo en tres surcos contiguos de líneas, flanqueados por dos surcos del probador. En la etapa de pre-floración, se eliminaron las espigas de la totalidad de las plantas de todas las líneas; la eliminación fue manual y antes de la liberación de polen. En la cosecha se obtuvieron al menos 17 mazorcas de cada mestizo (línea x probador).

La evaluación de mestizos se llevó acabo en tres localidades del estado de Morelos (Cuadro 2), en el ciclo de primavera-verano 2017. Se incluyeron los 15 mestizos, el probador VUAT, y las variedades comerciales H-515 y VS-535, liberadas por el Instituto Nacional de Investigaciones Forestales Agrícolas y Pecuarias (INIFAP); las tres últimas variedades se utilizaron como testigos. Los experimentos se establecieron bajo un diseño experimental de bloques completos al azar con cuatro repeticiones; la unidad experimental se conformó por dos surcos de $5 \mathrm{~m}$ de largo a una distancia entre surcos de $0.8 \mathrm{~m}$, y a una distancia entre plantas de $0.20 \mathrm{~m}$. Las variables medidas fueron: floración masculina (FM-días), altura de planta (AP-centímetros), peso de 100 semillas (P100S-gramos) y rendimiento de grano (RG-t ha ${ }^{-1}$ ); la totalidad de los datos de las variables fue probada para normalidad. El manejo agronómico de los experimentos se empleó de acuerdo con las especificaciones de la guía técnica del INIFAP para Morelos (Trujillo 2009).

El análisis estadístico de datos de las variables registradas se efectuó mediante análisis de varianza combinado y comparación de medias con la prueba de DMS (P $\leq$ 0.05). La estimación de la ACG se obtuvo con la siguiente formula: $g_{i}=\bar{X}_{m i}-\overline{\bar{X}}$; donde: $g_{i}$ es el efecto de la ACG; $\bar{X}$ es la media de cada variable en el i-ésimo mestizo, y $\overline{\bar{x}}$ es la media general de mestizos, de acuerdo con lo descrito por Chávez (1995). Los datos fueron procesados con el programa estadístico SAS (versión 9.1). 
Cuadro 1. Genealogía de líneas de maíz

\begin{tabular}{llcc}
\hline Línea & \multicolumn{1}{c}{ Genealogía } & $\begin{array}{c}\text { Nivel de } \\
\text { endogamia }\end{array}$ & $\begin{array}{c}\text { Criterio de selección } \\
\text { (tolerancia) }\end{array}$ \\
\hline${ }^{*}$ LUM13 & (T-43 x LPC15)-15-8 & $\mathrm{S}_{2}$ & Sequía \\
LUM40 & (Ac7643 x LPC15)-40-16 & $\mathrm{S}_{2}$ & Sequía \\
LUM73 & (Ac7643 x LPC15)-122-35 & $\mathrm{S}_{2}$ & Sequía \\
LUM76 & (Ac7643 x LPC15)-17-20 & $\mathrm{S}_{2}$ & Sequía \\
LUM80 & (Ac7643 x LPC15)-50-40 & $\mathrm{S}_{2}$ & Sequía \\
LUM121 & (T-43 x LPC15)-70-30 & $\mathrm{S}_{2}$ & Sequía \\
LUM124 & (Ac7643 x LPC15)-64-27 & $\mathrm{S}_{2}$ & Sequía \\
LUM148 & (T-43 x LPC15)-100-48 & $\mathrm{S}_{2}$ & Sequía \\
LUM194 & (T-43 x LPC15)-43-19 & $\mathrm{S}_{2}$ & Sequía \\
LUM209 & (T-43 x LPC15)-125-29 & $\mathrm{S}_{2}$ & Sequía \\
CML340 & LAPOSTASEQ-C3-FS20-4-1-1-2-3-B & $\mathrm{S}_{8}$ & Sequía \\
CML341 & LAPOSTASEQ-C3-FS1-2-2-2-1-1-B & $\mathrm{S}_{8}$ & Sequía y bajo N \\
CML342 & LAPOSTASEQ-C3-FS1-2-2-3-2-1-B & $\mathrm{S}_{8}$ & Sequía y bajo N \\
CML549 & (CML498/CLRCW36)-B-23-2-2-B*3-2-1 & $\mathrm{S}_{9}$ & Sequía y bajo N \\
CML576 & (CLFAWW11/CML494)-B-24-2-2-B-B-1-B-8-B-B & $\mathrm{S}_{11}$ & Sequía y bajo N \\
\hline
\end{tabular}

*LUM: Línea Universidad Autónoma del Estado de Morelos; CML: Línea de Maíz del CIMMyT; N: nitrógeno.

Cuadro 2. Sitios de evaluación de mestizos. Primavera-verano 2017

\begin{tabular}{lccccc}
\hline Localidad & Ubicación & $\begin{array}{c}\text { Altitud } \\
\mathbf{~ m}\end{array}$ & $\begin{array}{c}\text { T } \\
{ }^{\circ} \mathbf{C}\end{array}$ & $\begin{array}{c}\text { Pp } \\
\mathbf{~ m m}\end{array}$ & Suelo \\
\hline Tepalcingo & $18^{\circ} 37^{\prime} 46^{\prime \prime} \mathrm{N}$ y $98^{\circ} 51^{\prime} 00^{\prime \prime} \mathrm{O}$ & 1,100 & $13-34$ & 885 & Leptosol \\
Temoac & $18^{\circ} 46^{\prime} 37^{\prime \prime} \mathrm{N}$ y $98^{\circ} 47^{\prime} 14^{\prime \prime} \mathrm{O}$ & 1,580 & $10-31$ & 900 & Vertisol \\
Ayala & $18^{\circ} 44^{\prime} 29^{\prime \prime} \mathrm{N}$ y $98^{\circ} 54^{\prime} 38^{\prime \prime} \mathrm{O}$ & 1,220 & $10-32$ & 912 & Vertisol \\
\hline T: Temperatura mínima y máxima anual; Pp: Precipitación anual; Suelo predominante. Fuente: elaboración propia con datos de \\
INEGI (2017).
\end{tabular}

\section{Formación y evaluación de cruzas dialélicas}

En cuanto a las cruzas dialélicas, se formaron en el ciclo agrícola de primavera-verano 2017, en las mismas instalaciones que los mestizos. El procedimiento de formación fue: siembra de nueve líneas seleccionadas de la prueba de mestizos, con elección de cuatro líneas de ACG positiva para rendimiento de grano (LUM73, CML341, CML549, CML576) y cinco líneas de ACG negativa (LUM148, LUM80, LUM209, LUM76 y LUM 13), bajo el supuesto de que es posible identificar cruzas de buena ACE cuando interviene una línea de alta ACG con una de baja (Pérez-López et al. 2014; Cervantes-Ortiz et al. 2018). La siembra se hizo en un surco por línea de $5 \mathrm{~m}$ de largo, a una distancia entre surcos de $0.8 \mathrm{~m}$ y entre plantas de $0.25 \mathrm{~m}$, en dos repeticiones. Las cruzas se efectuaron de manera manual, con el objetivo de generar sólo cruzas directas. En la cosecha se obtuvieron al menos cinco mazorcas de cada una de las 36 cruzas.

La evaluación de campo de las cruzas dialélicas se condujo en el ciclo otoño-invierno 2017/2018, en el campo experimental de la EESuX, e incluyó las 36 cruzas dialélicas y los testigos H-515 y VS-535. La unidad experimental se conformó por dos surcos de $5 \mathrm{~m}$ de largo, a una distancia entre surcos de $0.8 \mathrm{~m}$ y entre plantas de $0.25 \mathrm{~m}$. Las variables de respuesta registradas fueron las mismas que en la evaluación de mestizos.

Los análisis estadísticos de las variables medidas se hicieron mediante análisis de varianza y comparación de medias, a través de la prueba de DMS (P $\leq 0.05)$. Se estimaron los efectos de la ACG y la ACE, utilizando 
el método IV de Griffing (1956), bajo el modelo 1 de efectos fijos; las fórmulas fueron las siguientes:

$g_{i}=\frac{1}{P(P-2)}\left[P X_{i .}-X_{. .}\right]$y $S_{i j}=X_{i j}-\frac{1}{P-2}\left(X_{i .}+X_{j .}\right)+\frac{1}{(P-1)(P-2)} X_{. .}$

Donde: $g_{i}$ es el efecto de la ACG en la i-ésima línea; $P$ es el número de líneas; $X_{i}$ es el total de la i-ésima línea a través de cruzas dialélicas; $X$.. es el gran total en el cuadro dialélico; $S_{i j}$ es el efecto de la ACE de la cruza $i \times j ; X_{i j}$ es el valor de la cruza $i \times j, y X_{j}$. es el total de la j-ésima línea a través de cruzas dialélicas. Los datos fueron procesados con el software SAS (versión 9.1).

\section{Evaluación de líneas per se}

La evaluación de campo con las líneas se estableció en el ciclo primavera-verano 2018; se incluyeron las 15 líneas endogámicas de maíz y los testigos H-515 y VS-535. El experimento se efectuó en el mismo campo experimental de la EESuX. El diseño experimental usado fue de bloques completos al azar con cuatro repeticiones; la unidad experimental se conformó por un surco de $5 \mathrm{~m}$ de largo a una distancia entre surcos de 0.8 $\mathrm{m}$, y entre plantas de $0.25 \mathrm{~m}$. Las variables estudiadas fueron las mismas que en la evaluación de mestizos y de cruzas dialélicas. El análisis estadístico de los datos consistió en análisis de varianza y comparación de medias por medio de la prueba de DMS $(\mathrm{P} \leq 0.05)$.

\section{Resultados Y Discusión}

\section{Evaluación de mestizos}

El análisis de varianza combinado a través de localidades (Cuadro 3) detectó diferencias estadísticas altamente significativas $(\mathrm{P} \leq 0.01)$ para todas las variables en las fuentes de variación de localidades y genotipos. Al descomponer la fuente de variación de genotipos, en mestizos, testigos y la comparación mestizos vs. testigos, se encontraron diferencias estadísticas ( $\mathrm{P} \leq$ 0.01) en todas las variables de respuesta en mestizos y testigos. En tanto que, en la comparación entre mestizos y testigos, sólo se identificaron diferencias estadísticas en las variables de peso de 100 semillas (P100S) y en rendimiento de grano (RG). Por otro lado, se reveló un comportamiento similar entre los mestizos provenientes de líneas de baja endogamia y entre los mestizos generados con líneas de alta endogamia, diferencias estadísticas encontradas únicamente en las variables de altura de planta (AP) y P100S; sin embargo, en la fuente de variación que compara mestizos formados con líneas de baja endogamia contra aquellos generados con líneas de alta, se identificaron diferencias estadísticas altamente significativas $(\mathrm{P} \leq$ 0.01 ) en todas las variables. En lo que se refiere a la

Cuadro 3. Cuadrados medios del análisis de varianza combinado a través de localidades en la evaluación de mestizos. Ciclo primavera-verano 2017

\begin{tabular}{lrrrrc}
\hline \multicolumn{1}{c}{ FV } & GL & $\begin{array}{c}\text { FM } \\
\mathbf{d}\end{array}$ & $\begin{array}{c}\text { AP } \\
\mathbf{~ m ~}\end{array}$ & $\begin{array}{c}\text { P100S } \\
\mathbf{g}\end{array}$ & $\begin{array}{c}\text { RG } \\
\text { tha }^{-1}\end{array}$ \\
\hline Localidades (L) & 2 & $861.90^{* *}$ & $51,298.25^{* *}$ & $112.79^{* *}$ & $337.61^{* *}$ \\
Rep/L & 9 & $2.12 \mathrm{~ns}$ & $1,388.45^{* *}$ & $3.48 \mathrm{~ns}$ & $1.04^{*}$ \\
Genotipos (G) & 17 & $6.09^{* *}$ & $1,926.66^{* *}$ & $25.35^{* *}$ & $2.04^{* *}$ \\
Mestizos & 14 & $5.12^{* *}$ & $1,576.69^{* *}$ & $15.19^{* *}$ & $1.18^{* *}$ \\
Baja endogamia & 9 & $1.56 \mathrm{~ns}$ & $1,053.74^{* *}$ & $10.38^{*}$ & $0.79 \mathrm{~ns}$ \\
Alta endogamia & 4 & $2.85 \mathrm{~ns}$ & $1,320.05^{* *}$ & $14.47^{* *}$ & $0.34 \mathrm{~ns}$ \\
Baja vs. alta & 1 & $46.22^{* *}$ & $7,309.81^{* *}$ & $61.38^{* *}$ & $7.97^{* *}$ \\
Testigos & 2 & $15.86^{* *}$ & $4,803.78^{* *}$ & $89.42^{* *}$ & $6.46^{* *}$ \\
Mestizos vs. testigos & 1 & $0.26 \mathrm{~ns}$ & $1,072.01 \mathrm{~ns}$ & $39.40^{*}$ & $5.24^{* *}$ \\
Interacción G L L & 34 & $3.38^{* *}$ & $210.74^{*}$ & $7.21^{*}$ & $0.83^{*}$ \\
Error & 153 & 1.34 & 129.92 & 4.60 & 0.48 \\
CV (\%) & & 1.74 & 4.40 & 7.65 & 14.41 \\
\hline
\end{tabular}

FV: Fuente de variación; GL: Grados de libertad; FM: Floración masculina; AP: Altura de planta; P100S: Peso de cien semillas; RG: Rendimiento de grano; CV: Coeficiente de variación; ns: No significativo; *: Significativo para P $\leq 0.05$; **: Significativo para P $\leq 0.01$. 
interacción genotipo $\times$ ambiente $(\mathrm{G} \times \mathrm{L})$, todas las variables mostraron diferencias estadísticas al $0.01 \mathrm{o}$ al 0.05 de probabilidad.

Las diferencias detectadas entre localidades se explican fundamentalmente por la altitud entre sitios $(1,100-1,550 \mathrm{~m})$, la cual influye en la temperatura ambiental, modificando la duración del ciclo biológico de las plantas, así como el tipo de suelo distinto entre localidades, lo que, a su vez, incideen el comportamiento del germoplasma, debido a diferencias en fertilidad y retención de humedad; en cuanto al comportamiento de los mestizos, se atribuye particularmente a la variabilidad entre grupos de líneas que difieren no sólo en el nivel de endogamia, sino también en el origen genético y geográfico de las líneas. La variación en la comparación de endogamia (baja vs. alta) se debe a que los mestizos de baja endogamia fueron un día más precoces, $13.5 \mathrm{~cm}$ más bajos y con menor peso de 100 semillas; en RG, los mestizos de alta endogamia superaron con $0.44 \mathrm{t} \mathrm{ha}^{-1}$ a los de baja endogamia. En cuanto a los testigos, el probador VUAT reflejó el menor rendimiento con $3.7 \mathrm{t} \mathrm{ha}^{-1}$, lo que confirma que es buen probador para evaluar la aptitud combinatoria de las líneas; esto coincide con la teoría que señala que un buen probador debe poseer frecuencias alélicas bajas para el carácter de interés (Hallauer et al. 2010; Lobato-Ortiz et al. 2010). En la interacción genotipo $x$ ambiente se encontró que el tipo de interacción en FM fue de cambio de rango, mientras que en el resto de variables fue de diferencia en magnitud, de acuerdo con los tipos de interacción descritos por Ferh (1991).

La estimación de la ACG de los mestizos (Cuadro 4) identificó a los mestizos formados con las líneas CML340, LUM73, CML341, CML342, CML549 y CML576 como los de mayor ACG para rendimiento de grano, con valores entre 0.579 y 0.114 t.ha ${ }^{-1}$; sólo la línea LUM73 es de baja endogamia. En cuanto al rendimiento de grano de dichos mestizos, éstos mostraron los mayores valores con una fluctuación entre 5.027 y 5.452 $\mathrm{t} \mathrm{ha}^{-1}$; el promedio de RG de los mestizos con ACG positiva resultó igual al RG del testigo H-515, y superó $14.1 \%$ el rendimiento del testigo VS-535, y $28.3 \%$ el rendimiento del probador. Los valores de ACG en FM indicaron que los mestizos integrados con las líneas LUM121, LUM76, LUM124 y LUM13 resultaron con efectos negativos y con valores que fluctuaron entre -1.0 y -0.5 d, líneas de baja endogamia. Respecto al efecto de la ACG en AP, el mestizo formado con la

Cuadro 4. Estimación de Aptitud Combinatoria General en cuatro variables, promedio de tres ambientes. Primavera-verano 2017

\begin{tabular}{lcccccccc}
\hline \multicolumn{1}{c}{ Mestizo } & ACG & RG & ACG & FM & ACG & AP & ACG & P100S \\
& RG & $\mathbf{t ~ h a}^{-1}$ & FM & d & AP & cm & P100S & g \\
\hline CML340 x VUAT & 0.539 & 5.452 & 1.1 & 67.5 & 12.8 & 272.7 & 1.7 & 29.5 \\
LUM73 x VUAT & 0.472 & 5.385 & -0.2 & 66.2 & 7.4 & 267.3 & 0.0 & 27.8 \\
CML341 x VUAT & 0.35 & 5.263 & 0.0 & 66.4 & -2.8 & 257.1 & 2.3 & 30.1 \\
CML342 x VUAT & 0.324 & 5.237 & 0.8 & 67.2 & 10.5 & 270.4 & 0.6 & 28.4 \\
CML549 x VUAT & 0.162 & 5.074 & 1.3 & 67.7 & 23.6 & 283.5 & -0.4 & 27.4 \\
CML576 x VUAT & 0.114 & 5.027 & 0.6 & 67.0 & 0.7 & 260.6 & 0.2 & 28.0 \\
LUM194 x VUAT & -0.044 & 4.868 & 0.1 & 66.5 & -4.7 & 255.2 & 1.1 & 28.9 \\
LUM148 x VUAT & -0.067 & 4.846 & 0.1 & 66.5 & -1.5 & 258.4 & 0.7 & 28.5 \\
LUM121 x VUAT & -0.131 & 4.782 & -1.0 & 65.4 & -6.2 & 253.7 & -0.7 & 27.1 \\
LUM80 x VUAT & -0.157 & 4.756 & -0.3 & 66.1 & 8.8 & 268.7 & -0.1 & 27.7 \\
LUM209 x VUAT & -0.208 & 4.705 & -0.3 & 66.1 & -10.6 & 249.3 & -0.2 & 27.6 \\
LUM76 x VUAT & -0.221 & 4.692 & -0.6 & 65.8 & -5.8 & 254.1 & -0.7 & 27.1 \\
LUM40 x VUAT & -0.247 & 4.666 & 0.1 & 66.5 & 1.3 & 261.2 & -1.0 & 26.8 \\
LUM13 x VUAT & -0.407 & 4.506 & -0.5 & 65.9 & -23.2 & 236.7 & -2.2 & 25.6 \\
LUM124 x VUAT & -0.479 & 4.434 & -0.6 & 65.8 & -10.9 & 249.0 & -0.6 & 27.2 \\
\hline
\end{tabular}

ACG: Aptitud combinatoria general; RG: Rendimiento de grano; FM: Floración masculina; AP: Altura de planta; P100S: Peso de cien semillas. 
línea LUM13 presentó el valor más bajo de $-23.2 \mathrm{~cm}$. Por otro lado, la ACG en P100S reportó que mestizos conformados por CML341 y CML340 fueron los que presentaron valores altos de 2.3 y $1.7 \mathrm{~g}$. la Cruz et al. 2009; Guzmán et al. 2017), lo que se ha asociado al tipo y origen de germoplasma de maíz utilizado, a las variables medidas y los alelos que las determinan y a los diferentes ambientes de evaluación.

\begin{tabular}{|c|c|c|c|c|c|}
\hline FV & GL & $\begin{array}{c}\text { FM } \\
\text { d }\end{array}$ & $\begin{array}{l}\mathrm{AP} \\
\mathrm{cm}\end{array}$ & $\begin{array}{c}\text { P100S } \\
\mathrm{g} \\
\end{array}$ & $\begin{array}{l}\text { RG } \\
\text { t ha-1 }\end{array}$ \\
\hline Bloques & 3 & $28.24^{* *}$ & $570.02^{* *}$ & $1.16 \mathrm{~ns}$ & $4.14^{* *}$ \\
\hline Cruzas & 35 & $6.10^{* *}$ & $698.27^{* *}$ & $12.10^{* *}$ & $3.95^{* *}$ \\
\hline ACG & 8 & $21.83^{* *}$ & $2,028.38^{* *}$ & $19.16^{* *}$ & $9.13^{* *}$ \\
\hline $\mathrm{ACE}$ & 27 & 1.44 & $304.16^{* *}$ & $10.01^{* *}$ & $2.41^{* *}$ \\
\hline Error & 105 & 2.87 & 118.55 & 4.07 & 0.78 \\
\hline CV (\%) & & 2.20 & 4.68 & 6.67 & 14.87 \\
\hline
\end{tabular}

FV: Fuente de variación; GL: Grados de libertad; FM: Floración masculina; AP: Altura de planta; P100S: Peso de cien semillas; RG: Rendimiento de grano; ns: No significativo; *: Significativo para $\mathrm{P} \leq 0.05 ; * *$ : Significativo para $\mathrm{P} \leq 0.01$.

Los mestizos con mayor ACG en el RG fueron los provenientes de las líneas CML340, LUM73, CML341, CML342, CML549 y CML576, con predominio de los mestizos de alta endogamia; estos resultados coinciden con lo encontrado en pruebas tardías, donde obtuvieron valores de 0.483 a 0.768 t.ha $^{-1}$ (Castañón et al. 1998). Al seleccionar líneas con alta ACG en rendimiento, éstas pueden usarse para generar cruzas con mayor potencial del que se usa comercialmente (Kamara et al. 2014), pero también existen reportes de que, al cruzar líneas de alta ACG con baja ACG o negativa, se ha encontrado buena ACE (Cervantes-Ortiz et al. 2018; Wong-Romero et al. 2006).

\section{Evaluación de cruzas dialélicas}

Los análisis de varianza para las cruzas dialélicas (Cuadro 5) detectaron diferencias estadísticas altamente significativas entre cruzas para todas las variables medidas, así como en la fuente de variación que prueban los efectos de ACG en todas las variables de respuesta; en cambio, para los efectos de ACE se encontraron diferencias estadísticas $(\mathrm{P} \leq 0.01)$ sólo en las variables de AP, P100S y RG. Los resultados encontrados en la ACG y la ACE indicaron que las líneas estudiadas difieren tanto en sus efectos genéticos aditivos, como entre los no aditivos en AP, P100S y RG. Resultados similares se han reportado en diferentes estudios (Rodríguez-Pérez et al. 2016; Guillen-de
Las estimaciones de los efectos de ACG de las líneas (Cuadro 6) revelaron que CML549 tuvo un efecto positivo de $1.81^{* *}$ días, en tanto que la línea que mostró el mayor valor negativo fue la LUM13 $\left(-1.02^{* *} \mathrm{~d}\right)$. En cuanto a la variable de altura de planta, la línea CML576 tuvo una ACG de 9.53** cm, y la LUM13 resultó con una ACG de $-15.36^{* *} \mathrm{~cm}$. En el comportamiento del P100S, la línea LUM148 presentó el mayor efecto positivo $\left(1.09^{* *} \mathrm{~g}\right)$ y LUM73 el mayor efecto negativo $\left(-1.03^{* *} \mathrm{~g}\right)$. En lo que respecta al RG, CML576 y CML341 mostraron la más alta y significativa ACG (1.14 y $0.45 \mathrm{t} \mathrm{ha}^{-1}$, respectivamente); este último resultado señala que ambas líneas tienen una contribución positiva e importante en la expresión del rendimiento del grano, lo cual las hace potencialmente adecuadas para incluirse en un programa de mejoramiento de maíz para generar variedades sintéticas (Guillen- de la Cruz et al. 2009).

Respecto a las estimaciones de los efectos de la ACE de las cruzas dialélicas (Cuadro 7), se encontró que las cruzas CML341 x LUM73, CML576 x LUM209 y CML549 x LUM76 manifestaron los mayores efectos positivos y estadísticamente significativos en la ACE, con valores de $1.03,0.82$ y $0.79 \mathrm{t} \mathrm{ha}^{-1}$, respectivamente. En la evaluación de mestizos, como en las cruzas dialélicas, las líneas CML341, CML576 y CML549 expresaron valores positivos en el efecto de la ACG, mientras que las líneas LUM73, LUM209 y LUM76 registraron valores negativos en la ACG, lo que 
demuestra que líneas endogámicas de alta endogamia y ACG positiva pueden generar híbridos aceptables en rendimiento de grano cuando se cruzan con líneas de baja endogamia y efectos negativos de ACG, de acuerdo con lo reportado en diferentes estudios (Guillen-de la Cruz et al. 2009; Cervantes-Ortiz et al. 2018), así como en el hecho mencionado de que al menos una de las líneas progenitoras se precisa que sea de alta ACG, para desarrollar híbridos sobresalientes (Pérez-López et al. 2014), debido a la aportación de genes con efectos aditivos.

\section{Evaluación per se}

Los resultados de la evaluación per se de líneas (Cuadro 8) revelaron que CML340 tuvo el mayor RG (5.04 tha-1) y la mayor AP $(263 \mathrm{~cm})$; esta línea, particularmente, provocó que las líneas de alta endogamia (CML) fueran superiores a las de baja endogamia (LUM). Otras sobresalientes de alta endogamia fueron CML342 y CML341, con RG de 2.64 y 2.18 t ha $^{-1}$, respectivamente; sin embargo, las LUM124 (2.43 $\left.\mathrm{t} \mathrm{ha}^{-1}\right)$ y LUM194 $\left(2.42 \mathrm{t} \mathrm{ha}^{-1}\right)$ produjeron rendimientos similares a las últimas; esto indica que las líneas del Programa de Mejoramiento de Maíz de la UAEM son competitivas en rendimiento de grano y es germoplasma con potencial para la formación de variedades sintéticas o híbridas, al coincidir en que líneas con bajos niveles de endogamia son vigorosas, rendidoras y pueden usarse como progenitores hembra en cruzas para aumentar la producción de semilla (Astate y Branco 2002). El método per se es considerado eficiente para conocer los aspectos agronómicos de las líneas (Rajaram et al. 1996; Ramírez et al. 1998).

Cuadro 6. Valores estimados de la ACG de líneas de maíz. Diseño IV de Griffing. Ciclo otoño-invierno 2017-2018

\begin{tabular}{|c|c|c|c|c|}
\hline Progenitores & $\begin{array}{c}\text { FM } \\
\text { d }\end{array}$ & $\begin{array}{l}\mathrm{AP} \\
\mathrm{cm}\end{array}$ & $\begin{array}{c}\text { P100S } \\
\text { g }\end{array}$ & $\begin{array}{l}\text { RG } \\
\text { t ha }^{-1}\end{array}$ \\
\hline CML576 & 0.06 & $9.53^{* *}$ & -0.36 & $1.14^{* *}$ \\
\hline CML341 & 0.56 & $5.06^{*}$ & $0.99^{* *}$ & $0.45^{* *}$ \\
\hline CML549 & $1.81^{* *}$ & $8.65^{* *}$ & $-1.35^{* *}$ & 0.22 \\
\hline LUM13 & $-1.02^{* *}$ & $-15.36^{* *}$ & 0.54 & 0.07 \\
\hline LUM209 & $-0.69^{*}$ & -1.58 & -0.09 & -0.08 \\
\hline LUM76 & $-0.94^{* *}$ & 1.86 & 0.07 & -0.20 \\
\hline LUM148 & 0.38 & $-11.36^{* *}$ & $1.09^{* *}$ & -0.22 \\
\hline LUM80 & 0.16 & $4.09^{*}$ & 0.14 & $-0.57^{* *}$ \\
\hline LUM73 & -0.30 & -0.89 & $-1.03^{* *}$ & $-0.80^{* *}$ \\
\hline
\end{tabular}

FM: Floración masculina; AP: Altura de planta; P100S: Peso de cien semillas; RG: Rendimiento de grano; *: Significativo para P $\leq 0.05$; **: Significativo para $\mathrm{P} \leq 0.01$.

Cuadro 7. Efectos de la ACE de 33 cruzas de maíz. Ciclo otoño-invierno 2017-2018

\begin{tabular}{lcccc}
\hline Progenitores & $\begin{array}{c}\text { FM } \\
\mathbf{d}\end{array}$ & $\begin{array}{c}\text { AP } \\
\mathbf{c m}\end{array}$ & $\begin{array}{c}\text { P100S } \\
\mathbf{g}\end{array}$ & $\begin{array}{c}\text { RG } \\
\mathbf{t h a}^{-1}\end{array}$ \\
\hline CML341 x LUM73 & 0.36 & 8.62 & -1.394 & $1.03^{* *}$ \\
CML576 x LUM209 & 0.00 & 5.69 & 0.960 & $0.82^{*}$ \\
CML549 x LUM76 & 0.50 & 2.95 & 1.448 & $0.79^{*}$ \\
LUM209 x LUM13 & -1.43 & -1.52 & 0.698 & 0.77 \\
CML341 x LUM76 & 0.25 & $16.56^{* *}$ & -0.004 & 0.74 \\
CML576 x LUM80 & -0.36 & -0.18 & 0.368 & 0.71 \\
CML549 x LUM209 & 0.75 & 7.65 & -1.033 & 0.62 \\
CML549 x LUM13 & -0.18 & 7.00 & 0.392 & 0.62 \\
\hline
\end{tabular}




\begin{tabular}{|c|c|c|c|c|}
\hline Progenitores & $\begin{array}{c}\text { FM } \\
\text { d }\end{array}$ & $\begin{array}{l}\mathrm{AP} \\
\mathrm{cm} \\
\end{array}$ & $\begin{array}{c}\text { P100S } \\
\mathrm{g} \\
\end{array}$ & $\begin{array}{l}\text { RG } \\
\mathbf{t} \mathbf{h a}^{-1}\end{array}$ \\
\hline LUM148 x LUM73 & -0.21 & 3.59 & -0.926 & 0.60 \\
\hline LUM80 x LUM76 & -0.86 & -8.32 & 0.990 & 0.46 \\
\hline LUM148 x LUM80 & -0.18 & 6.41 & 0.709 & 0.42 \\
\hline LUM80 x LUM13 & $1.46^{*}$ & $-14.35^{* *}$ & -0.321 & 0.38 \\
\hline CML341 x LUM13 & -0.18 & 11.34 & -0.235 & 0.37 \\
\hline LUM148 x CML341 & 0.18 & $-13.56^{* *}$ & -0.160 & 0.36 \\
\hline CML549 x LUM80 & 0.14 & 0.48 & $-1.990^{*}$ & 0.33 \\
\hline LUM148 x CML576 & 0.43 & 0.52 & -0.578 & 0.31 \\
\hline CML576 x LUM76 & 0.75 & 6.69 & 1.475 & 0.27 \\
\hline LUM148 x LUM209 & 0.18 & 2.96 & 1.181 & 0.23 \\
\hline LUM73 x LUM13 & -0.07 & -0.42 & -0.174 & 0.13 \\
\hline CML576 x LUM73 & 0.11 & -0.98 & 1.322 & 0.09 \\
\hline CML341 x LUM209 & 0.25 & -7.08 & $3.976^{* *}$ & -0.06 \\
\hline LUM73 x LUM80 & -0.50 & 0.56 & 1.720 & -0.15 \\
\hline LUM148 x CML549 & -0.32 & 7.40 & 0.722 & -0.17 \\
\hline LUM76 x LUM209 & -0.25 & -12.39 & -1.227 & -0.28 \\
\hline CML341 x LUM80 & 0.14 & $9.51^{*}$ & 1.036 & -0.29 \\
\hline LUM73 x LUM209 & 0.36 & -1.19 & $-2.037^{*}$ & -0.34 \\
\hline CML576 x LUM13 & 0.07 & 4.81 & 0.408 & -0.45 \\
\hline CML576 x CML549 & -0.75 & -5.19 & -0.977 & -0.53 \\
\hline LUM148 x LUM76 & -0.07 & -1.02 & -0.339 & -0.59 \\
\hline LUM73 x LUM76 & -0.64 & -3.91 & -0.190 & -0.62 \\
\hline CML549 x LUM73 & 0.61 & -6.27 & 1.680 & -0.74 \\
\hline LUM76 x LUM13 & 0.32 & -0.57 & -1.159 & $-0.76^{*}$ \\
\hline CML341 x CML549 & -0.75 & $-14.02^{* *}$ & -0.239 & $-0.91^{*}$ \\
\hline LUM148 x LUM13 & 0.00 & -6.30 & 0.391 & $-1.16^{* *}$ \\
\hline CML341 x CML576 & -0.25 & $-11.37^{*}$ & $-2.978^{* *}$ & $-1.23^{* *}$ \\
\hline LUM80 x LUM209 & 0.14 & 5.88 & $-2.516^{* *}$ & $-1.86^{* *}$ \\
\hline CML576 x LUM76 & 0.75 & 6.69 & 1.475 & 0.27 \\
\hline LUM148 x LUM209 & 0.18 & 2.96 & 1.181 & 0.23 \\
\hline LUM73 x LUM13 & -0.07 & -0.42 & -0.174 & 0.13 \\
\hline CML576 x LUM73 & 0.11 & -0.98 & 1.322 & 0.09 \\
\hline CML341 x LUM209 & 0.25 & -7.08 & $3.976^{* *}$ & -0.06 \\
\hline
\end{tabular}

FM: Floración masculina; AP: Altura de planta; P100S: Peso de cien semillas; RG: Rendimiento de grano; *: Significativo para $\mathrm{P} \leq 0.05$; **: Significativo para $\mathrm{P} \leq 0.01$. 
Cuadro 8. Características agronómicas y rendimiento de grano en

líneas de maíz tolerantes a sequía. Primavera-verano 2018

\begin{tabular}{lcccc}
\hline Línea & $\begin{array}{c}\text { FM } \\
\mathbf{d}\end{array}$ & $\begin{array}{c}\text { AP } \\
\mathbf{c m}\end{array}$ & $\begin{array}{c}\text { P100S } \\
\mathbf{g}\end{array}$ & $\begin{array}{c}\text { RG } \\
\mathbf{t} \mathbf{h a}^{-1}\end{array}$ \\
\hline LUM13 & 64 & 168 & 27.7 & 1.38 \\
LUM40 & 69 & 226 & 25.3 & 1.83 \\
LUM73 & 69 & 215 & 26.4 & 1.67 \\
LUM76 & 70 & 206 & 24.9 & 1.04 \\
LUM80 & 68 & 225 & 30.2 & 1.76 \\
LUM121 & 67 & 222 & 28.6 & 1.82 \\
LUM124 & 68 & 229 & 26.7 & 2.43 \\
LUM148 & 66 & 166 & 27.2 & 1.13 \\
LUM194 & 68 & 235 & 33.3 & 2.42 \\
LUM209 & 66 & 227 & 27.2 & 1.39 \\
CML340 & 64 & 263 & 34.5 & 5.04 \\
CML341 & 65 & 236 & 29.9 & 2.18 \\
CML342 & 68 & 251 & 27.8 & 2.64 \\
CML549 & 87 & 232 & 21.4 & 1.15 \\
CML576 & 70 & 207 & 23.6 & 1.48 \\
H-515 & 64 & 242 & 30.1 & 4.00 \\
VS-535 & 65 & 269 & 33.4 & 3.83 \\
DMS (0.05) & 2 & 16 & 5.2 & 0.70 \\
\hline
\end{tabular}

FM: Floración masculina; AP: Altura de planta; P100S: Peso de cien semillas; RG:

Rendimiento de grano.

\section{Conclusiones}

La evaluación de líneas a través de mestizos permitió determinar la ACG de líneas de maíz y seleccionar las líneas LUM73, CML341, CML549 y CML576 con ACG positiva. Las líneas CML341, CML549 y CML576, de alta ACG positiva en rendimiento de grano, formaron cruzas de alta ACE positiva cuando se cruzaron con las líneas LUM73, LUM209 y LUM76 de ACG negativa. Por su parte, las líneas CML341, CML576 y CML549 resultaron con valores positivos en el efecto de la ACG, tanto en la evaluación de mestizos como en cruzas dialélicas. Agronómicamente, las líneas CML340 (5.04 t ha-1 $)$, CML342 (2.64 t ha-1), CML341 (2.18 t ha-1), LUM124 (2.43 t ha-1) y LUM194 (2.42 $\left.\mathrm{t} \mathrm{ha}^{-1}\right)$ mostraron ser competitivas en rendimiento de grano.

\section{Agradecimientos}

Los autores agradecen al Centro Internacional de Mejoramiento de Maíz y Trigo y al Programa de Mejoramiento de Maíz de la Escuela de Estudios Superiores de Xalostoc, por facilitar el uso de las líneas de maíz evaluadas en esta investigación. 


\section{LITERATURA CITADA}

Akaogu CI, Badu-Apraku B, Adetimirin VO. 2017. Combining ability and performance of extra-early maturing yellow maize inbreds in hybrid combinations under drought and rain-fed conditions. The Journal of Agricultural Science 155: 1520-1540. https://doi. org/10.1017/S0021859617000636

Astate FA, de Miranda JB. 2002. Inbreeding depression in maize populations of reduced size. Scientia Agricola 59: 335-340. http://doi.org/10.1590/ S0103-90162002000200020

Bernardo R. 1992. Retention of genetically superior lines during early generations testcrossing of maize. Crop Science 32: 933-937. https://doi.org/10.2135/ cropsci1992.0011183X003200040019x

Buenrostro-Robles M, Lobato-Ortiz R, García-Zavala JJ, Sánchez-Abarca C. 2017. Rendimiento de líneas de maíz exótico irradiado con rayos gamma y de híbridos de cruza simple. Revista Fitotecnia Mexicana 40: 351-358.

Castañón G, Jeffers D, Hidalgo H, Tosquy H. 1998. Prueba de mestizos en maíz en el estado de Veracruz, México. Agronomía Mesoamericana 9: 89-96.

Cervantes-Ortiz F, Hernández-Esparza J, Gracia-Rodríguez JG, Rangel-Lucio JA, Andrio-Enríquez E, Mendoza-Elos M, Rodríguez-Pérez G, Rodríguez-Mercado D. 2018. Aptitud combinatoria general y especifica de caracteres agronómicos en líneas de maíz (Zea mays L.) de baja endogamia. Chilean Journal of Agricultural \& Animal Sciences 34: 33-42.

Chávez AJL. 1995. Mejoramiento de plantas 2. Métodos específicos de plantas alógamas. Editorial Trillas. Distrito Federal, México.

Escorcia-Gutiérrez N, Molina-Galán JD, Castillo-González F, Mejía-Contreras JA. 2010. Rendimiento, heterosis y depresión endogámica de cruzas simples de maíz. Revista Fitotecnia Mexicana 33: 271-279.

Falconer DS, Mackay TFC. 2006. Introducción a la genética Cuantitativa. Editorial Acribia, S. A. Zaragoza, España.

Ferh W. 1991. Principles of Cultivar Development: Theory and Technique. Iowa State University Press. Ames, USA.

Griffing B. 1956. Concept of general and specific combining ability in relation to diallel crossing systems. Australian Journal of Biological Sciences 9: 463-493.

Guillen-de la Cruz P, de la Cruz-Lázaro E, Castañón-Nájera G, Osorio-Osorio R, Brito-Manzano NP, Lozano-del Río A, López-Noverola U. 2009. Aptitud combinatoria general y específica de germoplasma tropical de maíz. Tropical and Subtropical Agroecosystems 10: 101-107.

Guzmán M, Díaz D, Ramis C, Figueroa-Ruiz R, Jiménez R. 2017. Estimación de la aptitud combinatoria y heterosis en híbridos no convencionales de maíz con alto contenido de proteína. Biagro 29: 175-184.

Hallauer RA, Carena JM, Miranda Filho JB. 2010. Quantitative Genetics in Maize Breeding. Handbook of Plant Breeding. Springer. New York, USA.

[INEGI]. Instituto Nacional de Estadística y Geografía. 2017. Anuario estadístico y geográfico de Morelos 2017. https://www.inegi.org.mx/app/publicaciones/default. html?t=0730003000000000\&ag=17. (Consultado el 30 de Agosto 2018).

Kamara MM, El-Degwy IS, Koyama H. 2014. Estimating ability of some maize inbred lines using line $x$ tester mating design under two nitrogen levels. Australian Journal of Crop Science 8: 1336-1342.

Ledesma-Miramontes A, Ramírez-Díaz JL, Vidal-Martínez VA, Peña-Ramos A, Ruiz-Corral JA, Salinas-Moreno Y, Preciado-Ortiz RE. 2015. Propuesta para integrar un patrón heterótico de maíz de grano amarillo para la zona de transición de México. II. Evaluación de mestizos y cruzas. Revista Fitotecnia Mexicana 38: 133-143.

Lobato-Ortiz R, Molina-Galán JD, López-Reynoso JJ, MejíaContreras JA, Reyes-López D. 2010. Criterios para elegir el mejor probador de la aptitud combinatoria general para rendimiento de grano de líneas autofecundadas de maíz. Agrociencia 44: 17-30.

López C, Tadeo-Robledo M, Espinosa-Calderón A, GarcíaZavala JJ, Benítez I, Vázquez MG, Carrillo JA. 2017. Productividad de cruzas simples de maíz con calidad de proteína en valles altos de México. Revista Mexicana de Ciencias Agrícolas 8: 559-570.

Pérez-López FJ, Lobato-Ortiz R, García-Zavala JdeJ, MolinaGalán JD, López-Reynoso JdeJ, Cervantes-Santana T. 2014. Líneas homocigóticas de maíz de alto rendimiento como progenitoras de híbridos de cruza simple. Agrociencia 48: 425-437.

Poehlman M, Sleper DA. 2003. Mejoramiento genético de las cosechas: Mejoramiento genético del Maíz. Limusa. México.

Rajaram S, Hans-Joachim B, Ginkel MV. 1996. CIMMYT Approach to breed for drought tolerance. Euphytica 92: 147-153.

Ramírez-Díaz JL, Vidal-Martínez VA, Alemán-de-la-Torre I, Ledesma-Miramontes A, Gómez-Montiel NO, Salinas- 
Moreno Y, Bautista-Ramírez E, Tapia-Vargas LM, RuizCorral A. 2019. Selección de líneas y cruzas de maíz combinando las pruebas de mestizos y cruzas dialélicas. Revista Fitotecnia Mexicana 42: 335-346.

Ramírez JL, Ron J, Sánchez J, Carcía A, Maya J. 1998. Aptitud combinatoria general y correlaciones fenotípicas entre líneas y mestizos de maíz. Agronomía Mesoamericana 9: 69-76.

Ramírez JL, Chuela M, Vidal VA, Ron J, Caballero F. 2007. Propuesta para formar híbridos de maíz combinando patrones heteróticos. Revista Fitotecnia Mexicana 30: 453-461.

Rebolloza HH, Castillo GA, Carapia RVE, Rodríguez AM, Villegas TOG, Núñez VME, Suárez RR, Perdomo RF. 2016. Estimación de parámetros genéticos y selección de líneas $\mathrm{S}_{1}$ en una población segregante de maíz tropical. Revista Mexicana de Ciencias Agrícolas 7: 1893-1904.

Rodríguez-Pérez G, Zavala-García F, Treviño-Ramírez JE, Ojeda-Zacarías C, Mendoza-Elos M, Rodríguez-Herrera SA, Cervantes-Ortiz F. 2016. Aptitud combinatoria y heterosis entre líneas de dos tipos de maíz para grano. Interciencia 41: 47-54.

[SAGARPA] Secretaría de Agricultura, Ganadería, Desarrollo Rural, Pesca y Alimentación. 2017. Boletín mensual. https://www.gob.mx/cms/uploads/attachment/file/214365/Bolet_nes_Balanzas_productos_agropecuarios.pdf. (Consultado el 16 de enero 2019).

Sánchez-Ramírez FJ, Mendoza-Castillo MC, MendozaRodríguez M, Castillo-González F, Cruz-Izquierdo S, Castro-Nava S, Molina-Galán JD. 2017. Aptitud combinatoria de líneas endogámicas para la producción de híbridos de maíz (Zea mays L.) de cruzas simples en condiciones de riego. Agrociencia 51: 393-407.

Sánchez-Ramírez FJ, Mendoza-Castillo MC, MendozaMendoza CG. 2020. Evaluación de mestizos y uso de técnicas multivariadas para identificar líneas sobresalientes de maíz. Revista Mexicana de Ciencias Agrícolas 11: 433-439. https://doi.org/10.29312/remexca.v11i2.1778

[SIAP] Servicio de Información Alimentaria y Pesquera. 2017. Anuario estadístico de la producción Agrícola. https://nube.siap.gob.mx/cierreagricola/. (Consultado el 22 de octubre 2018).

Sprague GE. 1946. Early testing of inbred lines of corn. Journal American Society of Agronomy 38: 108-117. https://doi.org/10.2134/agronj1946.000219620038000200 $02 x$

Trujillo CA. 2009. Guía para cultivar maíz bajo condiciones de riego en el Estado de Morelos. SAGARPA, INIFAP, CIRPAS. Campo Experimental Zacatepec. Folleto para productores No. 46. Zacatepec, Morelos, México. 17 pp. Velázquez-Cardelas GA, González-Huerta A, Pérez-López DJ, Castillo-González F. 2018. Comportamiento de mestizos en tres localidades del centro de México. Revista Mexicana de Ciencias Agrícolas 9: 1217-1230. https://doi. org/10.29312/remexca.v9i6.1586

Wong-Romero R, Gutiérrez-del Rio E, Rodríguez-Herrera SA, Palomo-Gil A, Córdova-Orellana H, EspinozaBanda A. 2006. Aptitud combinatoria y parámetros genéticos de maíz para forraje en la comarca Lagunera, México. Universidad y Ciencia Trópico Húmedo 22: 141-15. 